\title{
Sex differences in childhood hearing impairment and adult obesity
}

\section{Scott M Montgomery}

Clinical Epidemiology Unit, Örebro University Hospital, Sweden

scott.montgomery@ki.se

\section{Walter Osika}

Stress Research Institute, Stockholm University, Sweden.

\section{Ole Brus}

Biostatistics Unit, Örebro University Hospital, Sweden.

\section{Mel Bartley}

Department of Epidemiology and Public Health, University College London, UK

(Received April 2010 Revised September 2010)

\section{Abstract}

Some adult neurological complications of obesity may have early-life origins. Here, we examine associations of childhood hearing impairment with childhood and adult obesity, among 3288 male and 3527 female members of a longitudinal cohort born in Great Britain in 1970. Height and weight were measured at age 10 years and self-reported at 34 years. Audiometry was conducted at age 10 years. The dependent variable in logistic regression was minor bilateral hearing impairment as a marker of systemic effects, while $B M I$ at age 10 or 34 years were modelled as independent variables with adjustment for potential confounding factors including social class, maternal education and pubertal development at age 10 years. Among females, the adjusted odds ratios (and 95\% confidence intervals) for hearing impairment at age 10 years were 2.33 (1.36-3.98) for overweight/obesity; and at age 34 years they were 1.71 (1.00-2.92) for overweight and 2.73 (1.58-4.71) for obesity and the associations were not explained by Childhood BMI at age 10 years. There were no consistent associations among males and interaction testing revealed statistically significant effect modification by sex. The dose-dependent associations among females are consistent with childhood origins for some obesityassociated impaired neurological function and the possible existence of a 'pre-obese syndrome'. The accumulation of risks for poorer health among those who become obese in later life begins in childhood. Childhood exposures associated with bilateral hearing impairment are risks for obesity in later life among females.

Keywords: hearing impairment; cohort; obesity; audiometry; sex-difference

\section{Introduction}

Obesity is linked with adverse health sequelae, including type 2 diabetes mellitus and excess mortality. Obesity and type 2 diabetes are linked with neurological complications including more rapid cognitive decline and dementia in later life (Yaffe et al, 2004a). While obesity and diabetes can result in neurological damage, there may be a more complex pattern of cause and effect. There is evidence of impaired childhood neurological function among otherwise healthy children, who go on to develop obesity or diabetes in adulthood (Olsson et al, 2008, Osika and Montgomery, 2008, Chandola et al, 2006). These associations were independent of childhood body mass index (BMI), so contemporaneous obesity or overweight in childhood does not appear to be the explanation for 
poorer function. It is not clear if the neurological 'impairments' reflect genuine biological phenomena or are confounded by social factors, and if the subtle neurological impairments are themselves causally related with subsequent obesity; or if they result from early-life exposures increasing the risk of both childhood impairments and later obesity risk. This paper is concerned with the hypothesis that childhood exposures impair neurological development and also increase subsequent obesity risk.

The association of poorer childhood cognitive function with obesity and type 2 diabetes (Chandola et al, 2006, Olsson et al, 2008) might be explained by social confounding, as performance in tests of cognition is influenced by social and cultural factors (Tong et al, 2007). These factors could also increase obesity risk. For this reason, we then used physical control and coordination to indicate neurological function (Osika and Montgomery, 2008) and focused on hand control. The association of poorer coordination and greater clumsiness at ages 7 and 11 years, with later obesity, is consistent with the hypothesis that early exposures increase the risk of both impaired neurological development and subsequent obesity risk. However, it could be argued that poorer physical control and coordination could cause subsequent obesity by discouraging participation in sport and exercise.

There may be several non-mutually exclusive explanations for associations of impaired neurological function with obesity. More evidence is required to demonstrate that there is a biological basis for impaired neurological development in those who will be subsequently obese. Here we examine the association of adult BMI with another marker of childhood function, hearing impairment. Objective testing of hearing function may be less confounded by social factors than cognitive function testing, so is arguably a better marker of a biological mechanism. Unlike physical control and coordination, we hypothesise that hearing impairment is less likely to be a direct influence on physical exercise and unlikely to cause later obesity. However, there is some evidence of causation in the opposite direction: physical exercise may improve hearing ability (Cristell et al, 1998) suggesting that exercise may be a common pathway relevant both to obesity risk and hearing ability. By examining bilateral hearing impairment, we increase the likelihood of identifying a systemic phenomenon, which may involve neurological function. The aetiology of bilateral sensorineural hearing impairment in children is heterogeneous, and the cause can be unknown in over $40 \%$ of children with less severe hearing loss (Das, 1996). Over a third of children can have genetic or syndromal abnormalities, while other causes include infections and complications associated with premature birth (Das, 1996). Socioeconomic disadvantage is associated with a higher rate of hearing impairment (Egbuonu and Starfield, 1982) indicating a role for socially mediated exposures.

Although little is known about childhood hearing impairment and subsequent adult-onset obesity or diabetes, there is cross-sectional evidence from studies of adults. Hearing impairment in adults has been associated with contemporaneous adult waist circumference (Hwang et al, 2009), type 2 diabetes and impaired fasting glucose (Bainbridge et al, 2008). The latter study identified the most notable associations with minor hearing impairment in young adults (Bainbridge et al, 2008). The former study identified sex differences in associations with waist circumference (Hwang et al, 2009). Therefore, in this study, we examined minimal hearing impairment in children and stratified by sex.

Here, we examine whether bilateral hearing impairment at age 10 years is associated with $\mathrm{BMI}$ at ages 10 and 34 years among a general populationbased British birth cohort. This study is concerned with establishing whether an association exists between hearing and both contemporaneous and future BMI, rather than identifying specific causal processes. For this reason, the dependent variable in our statistical models (hearing impairment) should not necessarily be considered to be a direct consequence of, or temporally subsequent to, independent measures such as BMI. Our previous studies of physical control and coordination associated with adult obesity (Osika and Montgomery, 2008) and cognitive function with type 2 diabetes, were both performed using data from a 158 British birth cohort. To avoid a cohort-specific cluster of associations, this study uses a different birth cohort, following people who were born in 1970.

\section{Methods}

The 1970 British Cohort Study (BCS70) is following the lives of all residents in Great Britain born during one week in 1970 (CLS, 2009). The study collected information on all birth events in the target week with subsequent data collection follow-ups. Here, data from follow-ups at age 10 and 34 years are 
used for the main analysis. The original longitudinal sample consisted of 16,571, and 13,135 participated at age 10 years, with 9,316 at 34 years (CLS, 2009). Some 6,815 had complete data from the 10 and 34-year follow-ups. As this study was concerned with minor bilateral hearing impairment, cohort members with other types of hearing impairment were excluded, reducing the sample to 6,548. The 10-year follow-up was described as nationally representative (CLS, 2009). In the reduced sample analysed here, there was some loss of the most disadvantaged families compared with the broader group of responders. Compared with the entire population available at age 10 years, the proportion in parental social class $\mathrm{V}$ (the most disadvantaged class based on occupation) dropped from $4.0 \%$ to $3.6 \%$. The most notable loss was from the relatively small group who could not be assigned a social class, from $11.1 \%$ to $2.5 \%$ ( $n=175$ among those included in the analysed sample).

Community medical officers and school nurses conducted an examination and medical record review at age 10 years. This included measurement of height and weight, which was used to calculate $\mathrm{BMI}$, classified using the age- and sex-standardised Cole criteria (Cole et al, 2000). The overweight and obese categories at age 10 years were combined due to small numbers. Sweep and pure-tone audiometry identified minimal, moderate or marked hearing impairment. A pubertal development score was based on the summed total number of pubertal signs identified during the examination among: breast development, pubic hair, axillary hair, testicular enlargement, menarche, penile enlargement and a final category, 'other signs of puberty'. All chronic illnesses and disabilities were recorded from examination and medical record review and parents reported childhood infections. Parents' occupations were used to estimate the Registrar General's social class. Father's occupation was used, but where no father was present, mother's occupation was used to estimate social class. Mothers reported their educational qualifications. At age 34 years, cohort members reported their height and weight and the resulting $\mathrm{BMI}$ was categorised (table 1) using the 1995 WHO criteria (WHO, 1995).

A subset of the cohort members included in the analysis also had complete information on gestational age and birth weight $(n=5,141)$. This information was recorded by midwives at the time of the birth.

\section{Statistical analysis}

Minor bilateral hearing impairment at age 10 years (our marker of systemic influences on function) was the dependent variable in logistic regression analysis. Although temporally preceding adult $\mathrm{BMI}$, this model was chosen so that BMI at ages 10 and 34 years could be modelled simultaneously as independent variables, with the possibility to assess patterns of association across BMI categories. The minority of cohort members with more severe and unilateral hearing impairments were excluded from the analysis. Associations of hearing impairment with BMI at ages 10 and 34 years were examined with and without mutual adjustment for each other, and with adjustment for the puberty score, parental social class, whether mother and father lived in the household and highest maternal educational qualification. All measures were modelled as a series of binary dummy variables.

As there were fewer valid data for the measures of gestational age and birth weight, and also because these measures may have a complex pattern of association with obesity, a separate set of models was adjusted for these factors and for the puberty score, parental social class, whether mother and father lived in the household and highest maternal educational qualification. These measures were modelled as continuous and categorical variables, but neither strategy was markedly more effective than the other in altering the association of BMI with hearing.

The analyses were stratified by sex. Interaction testing (including an interaction term and the main effects in the model) estimated effect modification.

To ensure that the results were not due to childhood chronic illness or disability, the analyses were repeated excluding cohort members who had relevant conditions identified by the examination and medical record review conducted at age 10 years: these were syndromes or conditions defined in the examination as resulting in mental/development retardation, as well as epilepsy and diabetes mellitus.

Further models were adjusted for history of mumps, measles, chickenpox, meningitis and recurrent ear infections.

SPSS version 15 and PASW Statistics version 18 (PASW, 2009) software packages were used. 


\section{Results}

Table 1 shows the characteristics of the sample by sex. Girls were more likely to be overweight or obese at age 10 years, but more men had a BMI

were no other sex differences, except for a larger number of pubertal development signs by age 10 years among girls. above the normal category at age 34 years. There

Table 1. Study population characteristics by sex

$\mathrm{BMI}$ at age 10 years

Underweight ( $<14.64$ for boys, $<14.61$ for girls)

Normal (14.64 to 19.84 for boys, 14.61 to 19.86 for girls)

Overweight or obese ( $>19.84$ for boys, $>19.86$ for girls)

$\mathrm{BMI}$ at age 34 years

Underweight $(<18.5)$

Normal $(18.5$ to $<25)$

Overweight $(25$ to $<30)$

Obese $(>30)$

Signs of puberty at age 10 years

None

One

Two or more

Parental social class (Registrar General's)

I

II

III non-manual

III manual

IV

$\mathrm{V}$

Not employed

Highest level of maternal qualifications

None

O level or equivalent

A level or equivalent

University degree or equivalent

Other qualifications

Hearing impairment at age 10 years

None

Minimal bilateral

Moderate or marked bilateral

Unilateral

Total

Associations of the potential confounding factors with minor bilateral hearing impairment are presented in table 2. Among the childhood factors, only parental social class is weakly associated with hearing loss in girls, not boys, and this association was further attenuated in the adjusted model.

$\begin{array}{ll}\text { Males } & \text { Females } \\ \mathbf{n}(\%) & \mathbf{n}(\%) \\ 330(10.0) & 393(11.1) \\ 2730(83.0) & 2730(77.4) \\ 228(6.9) & 404(11.5) \\ & \\ 23(0.7) & 83(2.4) \\ 1294(39.4) & 2025(57.4) \\ 1425(43.3) & 883(25.0) \\ 546(16.6) & 536(15.2) \\ & \\ 3148(95.7) & 2608(73.9) \\ 125(3.8) & 673(19.1) \\ 15(0.5) & 246(7.0) \\ & \\ 231(7.0) & 220(6.2) \\ 830(25.2) & 892(25.3) \\ 368(11.2) & 390(11.1) \\ 1295(39.4) & 1374(39.0) \\ 378(11.5) & 418(11.9) \\ 115(3.5) & 129(3.7) \\ 71(2.2) & 104(2.9) \\ 1588(48.3) & 1743(49.4) \\ 346(10.5) & 359(10.2) \\ 76(2.3) & 83(2.4) \\ 63(1.9) & 61(1.7) \\ 1215(37.0) & 1281(36.3) \\ 3086(93.8) & 3306(93.7) \\ 109(3.3) & 119(3.4) \\ 77(2.3) & 82(2.3) \\ 19(0.6) & 20(0.6) \\ 3288 & 3527 \\ & \end{array}$

Among the prenatal measures, low birth weight and premature birth were both associated with higher risk of hearing loss in boys but not girls.

Table 3 shows the association of BMI at ages 10 and 34 years with minor hearing impairment at age 10 years. Among males there is no evidence of an 
association with $\mathrm{BMI}$ at age 10 years. There was no consistent pattern of association between hearing impairment at age 10 years and BMI at age 34 years among males. A statistically significant inverse association was observed with the overweight category at age 34 years and this remained in the adjusted models. This isolated association may be due to chance, as there is no consistency in the pattern of association.

Among females there are statistically significant dose-dependent positive associations of hearing impairment at age 10 years with higher BMI at ages 10 and most notably 34 years, independent of the potential confounding factors (table 3 ). The associations remain after mutual simultaneous adjustment for BMI at both ages. There is no association with the underweight category.

The magnitude of the statistically significant associations between $\mathrm{BMI}$ and hearing impairment among females was not attenuated by additional adjustment for birth weight and gestational age. The adjusted odds ratios are 2.81 (1.45-5.47) for obesity at 34 years and 3.18 (1.74-5.82) for overweight/obesity at age 10 years. There was no notable change for the results among males.

Stratification by sex revealed that the magnitude of association between obesity and hearing impairment was much greater among women than men (table 3). This sex difference was quantified through the interaction of sex with BMI at age 34 years for associations with hearing impairment. Interaction testing produced statistically significant odds ratios of $3.72(1.75-7.92)$ for overweight and 2.87 (1.30-6.31) for obesity. The interaction of sex with $\mathrm{BMI}$ at age 10 years for the association with hearing impairment also produced a statistically significant odds ratio of $3.36(1.07-10.50)$ for the combined overweight/obesity category in children.

Exclusion of childhood chronic illness and disability at age 10 years, or adjustment for the infections, did not alter the main findings notably. The models were additionally adjusted for region of residence, and there was nothing to suggest that regional variation accounted for the findings. Exclusion of the minority of cohort members with an ethnic background other than the indigenous British population did not alter the findings notably (data not shown).

\section{Discussion}

Minor bilateral hearing impairment at age 10 years was positively associated with overweight and obesity, both contemporaneously at age 10 years and subsequently at age 34 years in female cohort members, but not males. The associations were independent of markers of family circumstances in childhood and pubertal development, as well as birth weight and gestational age. BMI at age 10 years did not appear to explain the association of childhood hearing with future overweight and obesity. The lack of positive associations among males was unexpected. The study benefited from detailed prospectively collected measures at birth and age 10 years, although measures at age 34 years were self-reported. The study used bilateral hearing impairment in childhood as a marker of a systemic, possibly neurological, impairment to examine the hypothesis that early life exposures may impair neurological function in those who will subsequently become obese. Previous studies of contemporaneous hearing impairment and suboptimal insulin signalling, or obesity in adulthood, identified associations with minor rather than more severe hearing impairment (Bainbridge et al, 2008), so this study also focused on minor impairment.

The number of male cohort members with minor hearing impairment was relatively small, so it is possible that the observed sex difference is a chance finding. It is less likely that the findings among females are due to chance, as dosedependent associations were observed for both childhood and adult BMI. Also, interaction testing identified statistically significant effect modification of the association between hearing and BMI (both in childhood and adulthood) by sex. Differential reporting bias by sex for the self-reported anthropometric data at age 34 years is unlikely to account for the results, as statistically significant associations with measured $\mathrm{BMI}$ at age 10 years among females were also observed. It is notable that, while the majority of women were in the 'normal' BMI category at age 34 years, a slightly higher proportion of males were in the overweight compared with the 'normal' category. Thus, overweight and obesity in women may represent a more extreme phenomenon with stronger links to adverse exposures such as those associated with childhood hearing impairment. 


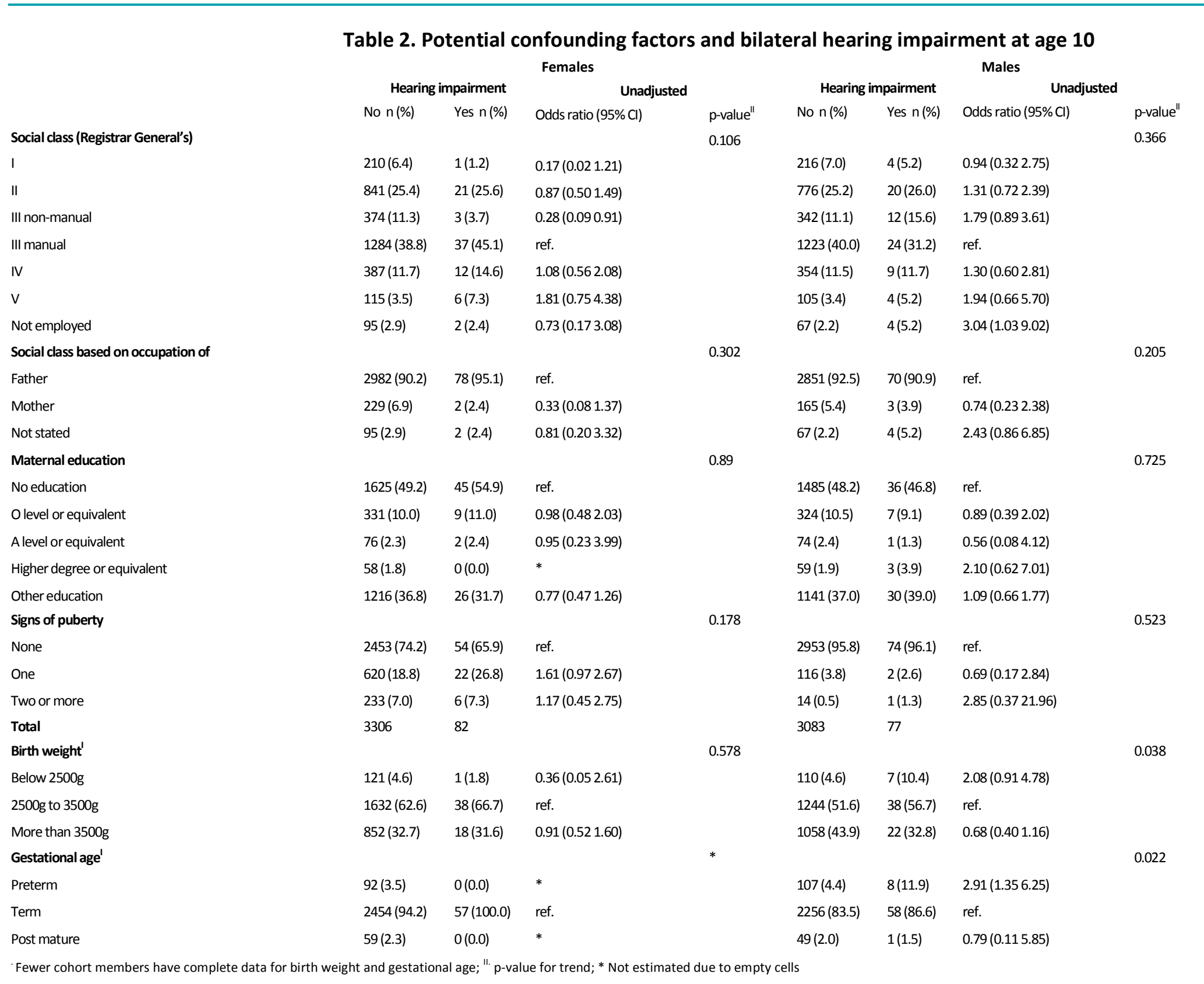


Table 3. Bilateral hearing impairment at age 10 years and BMI

\begin{tabular}{|c|c|c|c|c|c|c|c|}
\hline \multirow[t]{2}{*}{ MALES } & \multicolumn{2}{|c|}{ Hearing impairment } & \multicolumn{2}{|c|}{ Unadjusted } & \multicolumn{2}{|l|}{ Adjusted' } & Adjusted" \\
\hline & No, $n(\%)$ & Yes, n (\%) & Odds ratio $(95 \% \mathrm{Cl})$ & p-value ${ }^{\prime \prime \prime}$ & Odds ratio $(95 \% \mathrm{Cl})$ & p-value ${ }^{\prime \prime \prime}$ & Odds ratio $(95 \% \mathrm{Cl}) \mathrm{p}$-value ${ }^{\prime \prime \prime}$ \\
\hline BMI at age 34 years & & & & 0.041 & & 0.051 & 0.049 \\
\hline Underweight & $22(0.7)$ & $1(1.3)$ & 1.44 (0.19 10.98) & & $1.26(0.169 .80)$ & & $1.13(0.149 .08)$ \\
\hline Normal & $1206(39.1)$ & $38(49.4)$ & ref. & & ref. & & ref. \\
\hline Overweight & $1352(43.9)$ & $21(27.3)$ & $0.49(0.290 .84)$ & & $0.50(0.290 .86)$ & & $0.52(0.300 .90)$ \\
\hline Obese & $503(16.3)$ & $17(22.1)$ & 1.07 (0.60 1.92) & & $1.08(0.601 .95)$ & & $1.19(0.642 .21)$ \\
\hline BMI at age 10 years & & & & 0.605 & & 0.595 & 0.621 \\
\hline Underweight & $307(10.0)$ & $10(13.0)$ & 1.33 (0.67 2.61) & & $1.32(0.672 .60)$ & & $1.21(0.602 .46)$ \\
\hline Normal & $2564(83.2)$ & $63(81.8)$ & Ref & & Ref. & & ref. \\
\hline Overweight/Obese & $212(6.9)$ & $4(5.2)$ & 0.77 (0.28 2.13) & & 0.74 (0.27 2.07) & & 0.65 (0.23 1.87) \\
\hline Total & 3083 & 77 & & & & & \\
\hline \multicolumn{8}{|l|}{ FEMALES } \\
\hline BMI at age 34 years & & & & 0.001 & & 0.004 & 0.033 \\
\hline Underweight & $79(2.4)$ & $0(0.0)$ & $*$ & & $*$ & & $*$ \\
\hline Normal & $1921(58.1)$ & $32(39.0)$ & ref. & & ref. & & ref. \\
\hline Overweight & $818(24.7)$ & $25(30.5)$ & 1.83 (1.08 3.12) & & 1.71 (1.00 2.92) & & 1.65 (0.96 2.83) \\
\hline Obese & $488(14.8)$ & $25(30.5)$ & 3.08 (1.81 5.24) & & 2.73 (1.58 4.71) & & 2.37 (1.33 4.24) \\
\hline BMI at age 10 years & & & & 0.002 & & 0.008 & 0.116 \\
\hline Underweight & $371(11.2)$ & $8(9.8)$ & 1.03 (0.48 2.17) & & $1.08(0.512 .30)$ & & $1.31(0.612 .81)$ \\
\hline Normal & $2567(77.6)$ & $54(65.9)$ & ref. & & ref. & & ref. \\
\hline Overweight/Obese & $368(11.1)$ & $20(24.4)$ & 2.58 (1.53 4.37) & & 2.33 (1.36 3.99) & & 1.79 (1.02 3.14) \\
\hline Total & 3306 & 82 & & & & & \\
\hline
\end{tabular}


Although not an a priori hypothesis, the sex difference in association with hearing impairment is consistent with previously observed greater femalespecific susceptibility to early life socio-economic risks for metabolic syndrome (Chichlowska et al, 2009) and obesity (Khlat et al, 2009). Do such socially mediated exposures increase the risk of both hearing impairment and obesity in females? Disadvantage is certainly associated with hearing impairment (Egbuonu and Starfield, 1982) and although we identified a weak association with social class among girls, this measure could not explain associations between hearing and BMI. The sex difference could lie in greater weight gain susceptibility among females exposed to risks for hearing impairment. A possible alternative explanation for the sex difference is selection bias: a higher proportion of disadvantaged males than females (with greater obesity and hearing loss risk) could have been lost to follow-up. This might help to explain the inconsistency of our findings with studies showing cross-sectional associations of hearing loss with obesity among adult males (Hwang et al, 2009).

We can only speculate about the specific mechanisms underlying hearing loss. This may be sensorineural hearing impairment, involving abnormalities of the central nervous or auditory systems. These findings add to the earlier evidence of poorer neurological function associated with subsequent obesity (Olsson et al, 2008, Osika and Montgomery, 2008, Chandola et al, 2006). Influences on neurological function could include hormones such as oestrogen, which may be implicated as indicated by menstrual fluctuation in auditory perception (Haggard and Gaston, 1978), perhaps acting through inner-ear oestrogen receptors (Stenberg et al, 2001). Perhaps hormonal effects on hearing could be reversible. Growth hormones could also be relevant, as IGF1 is implicated in the development of childhood hearing (Welch and Dawes, 2007). However, this study did not reveal any evidence of hormonal involvement, as neither height at age 10 years, nor pubertal development among girls, explained the associations of BMI with hearing impairment.

We speculate that one relevant exposure may be psychosocial stress in girls. Significant exposure (or susceptibility) to stress, results in chronic activation of glucocorticoid receptors, and this may damage hearing through detrimental effects on the central nervous system: the influence of the hypothalamic-pituitary-adrenal (HPA) axis - which is central to the stress response - on the auditory system has been demonstrated (Welch and Dawes, 2007). Psychosocial stress is also associated with childhood obesity (Dockray et al, 2009), adult type 2 diabetes (Eriksson et al, 2008) and weight gain (Fowler-Brown et al, 2009). We recently demonstrated that maternal stress, likely to be a potent source of chronic stress for young children, is associated with overweight in three-year-olds; and this association was independent of markers of socio-economic circumstances (Stenhammar et al, 2010). Some of the putative effects of stress on weight gain could be through metabolic and behavioural influences, as suggested by animal models (Alsio et al, 2009). Another aspect of the influence of maternal stress could operate through maternal behaviour, as mothers with higher stress levels, on average left their three-year-olds watching television for notably longer periods each day (Stenhammar et al, 2010). This will influence physical activity, but might also represent inadequate stimulation relevant to neurological development and function. The beneficial effect of exercise on hearing ability (Cristell et al, 1998) may also be relevant in this context. While exposures other than stress will be important influences on weight gain in children, associations with another measure lend support to a causal role for stress. In the earlier study, some forms of non-secure parental attachment style were also associated with overweight in offspring. Attachment style indicates personal characteristics relevant to interpersonal interactions, and is thought to be stable over time. Some non-secure attachment styles in parents could result in stressful exposures among offspring, and in our earlier study, influences on children's weight associated with parental attachment style appeared to operate through stress. There may be sex-specific physiological responses to some types of stress with greater susceptibility among girls (Osika et al, 2009), although the lack of disparity by sex in proportion with hearing impairment suggests that the difference lies in susceptibility to risks for weight gain.

It has been suggested that developmental coordination disorder (DCD) could explain the association of obesity with poorer physical control and coordination, signalling impaired neurological function, as poorer coordination may limit 
participation in physical exercise and thus increase obesity risk (Cairney et al, 2010). DCD is associated with impaired foetal development, indicated by markers such as low birth weight (Holsti et al, 2002). As hearing impairment can also have early life origins signalled by low birth weight (Cristobal and Oghalai, 2008) and premature birth (Leversen et al, 2010), exposures in utero may be relevant to poorer neurological development, hearing impairment and obesity risk. For this reason we additionally adjusted for birth weight and gestational age, but limited this to sub-analyses for two reasons. The first reason is that not all of the subjects included in the main analyses had full data for birth weight and gestational age, and we wished to include the maximum number. The second reason is that associations of birth weight with $D C D$ and obesity may be contradictory, making interpretation of the results potential problematic: DCD is associated with low birth weight (Holsti et al, 2002), while higher birth weight is a significant risk for subsequent obesity even though this may be due to confounding (The et al, 2010). Only a minority of people with poorer physical control and coordination coupled with obesity are likely to also have DCD (Montgomery, 2010).

Potential limitations of this study include the lack of glycaemic status measurements and the use of $\mathrm{BMI}$, rather than more precise measures of adipose tissue distribution relevant to metabolic influences, as these measures were not available. The adult anthropometric measures were selfreported so possibly imprecise, introducing error and possibly bias. The loss of participants from the most disadvantaged families may have biased the results, and have excluded a notable proportion that became obese. Hearing was only measured in childhood, so it is not known if the hearing impairment in females persists into adulthood, nor whether males develop impairments at later ages. Hearing impairment was precisely measured in childhood but the causes may be somewhat heterogeneous. To tackle this, we only considered minor hearing impairment, as previous studies found this was most notably associated with contemporaneous obesity and related measures. We only considered bilateral hearing loss to identify systemic problems, but in a proportion of those with bilateral impairment, there may be nonsystemic influences that affect both ears. Such considerations could reduce the observed associations or may conceivably inflate them.

Confounding in a study such as this is a possibility, so we adjusted for parental social class (and if based on father's or mother's occupation), which indicates cultural and material circumstances in childhood; and is relevant both to obesity and hearing loss risk (Khlat et al, 2009, Egbuonu and Starfield, 1982). We also adjusted for maternal education as this is another marker of factors relevant to obesity risk (Stenhammar et al, 2010). A measure of pubertal signs was included, as development at age 10 years is likely to be relevant to childhood BMI and potentially to other aspects of development. We additionally adjusted for birth weight and gestational age, as markers of foetal development potentially relevant to weight gain and neurological development. However, the association of hearing impairment with obesity in women over 20 years later is independent of these markers of family circumstances and foetal development; and it could not be explained by childhood chronic illness or disability. We also adjusted for childhood BMI, which clearly represents over-adjustment, but also provides useful information. Adjustment for childhood BMI not only indicates that childhood body mass is associated with contemporaneous hearing impairment, but also that the association of hearing with adult BMI is not explained by childhood BMI itself. Adjustment for childhood BMI also represents indirect adjustment for a variety of factors, such as physical activity, that are plausible confounding factors. However, BMI may be too crude a measure to identify specific types of adipose tissue in childhood that indicate exposures or produce bioactive compounds (Knecht et al, 2008, Yaffe et al, 2004b) relevant to neurological function.

Minor hearing impairment in girls is added to the measures of poorer childhood function associated with obesity in adulthood. This association provides more evidence of poorer neurological function among those who will subsequently become obese or develop type 2 diabetes, indicating that some neurological complications, or susceptibility to them, that are associated with these diseases have their origins in early life. As poorer childhood function and development predicts poorer adult function (Kuh et al, 2006a, Kuh et al, 2006b) this is also likely to be 
associated with more rapid limitation with decline in old age and thus poorer health. Early life exposures impairing development and function are associated with accumulation of later risks: those with poorer childhood function are also more likely to be exposed to the risks coupled with adult obesity. Accumulation of risks could occur through a variety of mechanisms that are not mutually exclusive. These include lower-level educational attainment associated with poorer cognitive function leading to labour market disadvantage and behavioural risks; lower levels of physical activity due to poorer physical control and coordination (Olsson et al, 2008, Osika and Montgomery, 2008, Chandola et al, 2006). It should be stressed that the previous study of neurological function indicated by physical control and coordination associated with later obesity, found that the association could not be explained by lower cognitive function, indicating that the problems described are not due to lower intelligence.

Further research should identify relevant risks, mechanisms and whether a 'pre-obese syndrome' associated with a range of impairments exists. While the childhood impairments pre-dating obesity may be relatively minor, they are worthy of further investigation and could signal other developmental effects and susceptibility to more rapid functional decline in later life, further accelerated by adult obesity. Understanding the accumulation of risks over life is likely to be key in identifying how early exposures impairing childhood function are related to more severe adverse outcomes in later life. When sorrows come, they come not as single spies, but in battalions Hamlet, Act IV scene V.

\section{Acknowledgement}

This study received support from Economic and Social Research Council grant RES - 596-28-0001 to the International Centre for Life Course Studies in Society and Health.

\section{References}

Alsio J, Roman E, Olszewski PK, Jonsson P, Fredriksson R, Levine AS, Meyerson BJ, Hulting AL, Lindblom J and Schioth HB. (2009) Inverse association of high-fat diet preference and anxiety-like behavior: a putative role for urocortin 2. Genes Brain and Behavior, 8, 193-202.

Bainbridge KE, Hoffman HJ and Cowie CC. (2008) Diabetes and hearing impairment in the United States: audiometric evidence from the National Health and Nutrition Examination Survey, 1999 to 2004. Annals of Internal Medicine, 149, 1-10.

Cairney J, Hay J, Veldhuizen S, Missiuna C, Mahlberg N and Faught BE. (2010) Trajectories of relative weight and waist circumference among children with and without developmental coordination disorder. Canadian Medical Association Journal, 182, 1167-72.

Chandola T, Deary IJ, Blane D and Batty GD. (2006) Childhood IQ in relation to obesity and weight gain in adult life: the National Child Development (1958) Study. International Journal of Obesity (London), 30, 1422-32.

Chichlowska KL, Rose KM, Diez-Roux AV, Golden SH, McNeill AM and Heiss G. (2009) Life course socioeconomic conditions and metabolic syndrome in adults: the Atherosclerosis Risk in Communities (ARIC) Study. Annals of Epidemiology, 19, 875-83.

Centre for Longitudinal Studies. (2009) The 1970 British Cohort Study. http://www.cls.ioe.ac.uk/studies.asp?section $=000100020002$

Cole TJ, Bellizzi MC, Flegal KM and Dietz WH. (2000) Establishing a standard definition for child overweight and obesity worldwide: international survey. British Medical Journal, 320, 1240-3.

Cristell M, Hutchinson KM and Alessio HM. (1998) Effects of exercise training on hearing ability. Scandinavian Audiology, 27, 219-24.

Cristobal R and Oghalai JS. (2008) Hearing loss in children with very low birth weight: current review of epidemiology and pathophysiology. Archives of Disease in Childhood Fetal and Neonatal Edition, 93, F462-8.

Das, VK. (1996) Aetiology of bilateral sensorineural hearing impairment in children: a 10 year study. Archives of Disease in Childhood, 74, 8-12.

Dockray S, Susman EJ and Dorn LD. (2009) Depression, cortisol reactivity, and obesity in childhood and adolescence. Journal of Adolescent Health, 45, 344-50.

Egbuonu L and Starfield A. (1982) Child health and social status. Pediatrics, 69, 550-7.

Eriksson AK, Ekbom A, Granath F, Hilding A, Efendic S and Ostenson CG. (2008) Psychological distress and risk of pre-diabetes and Type 2 diabetes in a prospective study of Swedish middle-aged men and women. Diabetic Medicine, 25, 834-42. 
Fowler-Brown AG, Bennett GG, Goodman MS, Wee CC, Corbie-Smith GM and James SA. (2009) Psychosocial Stress and 13-year BMI Change Among Blacks: the Pitt County Study. Obesity (Silver Spring), 17, 2106-9.

Haggard M and Gaston JB. (1978) Changes in auditory perception in the menstrual cycle. British Journal of Audiology, 12, 105-18.

Holsti L, Grunau RV and Whitfield MF. (2002) Developmental coordination disorder in extremely low birth weight children at nine years. Journal of Developmental and Behavioral Pediatrics, 23, 9-15.

Hwang JH, Wu CC, Hsu CJ, Liu TC and Yang WS. (2009) Association of central obesity with the severity and audiometric configurations of age-related hearing impairment. Obesity (Silver Spring), 17, 1796801.

Khlat M, Jusot F and Ville I. (2009) Social origins, early hardship and obesity: a strong association in women, but not in men? Social Science and Medicine, 68, 1692-9.

Knecht S, Ellger T and Levine JA. (2008) Obesity in neurobiology. Progress in Neurobiology, 84, 85-103.

Kuh D, Hardy R, Butterworth S, Okell L, Richards M, Wadsworth M, Cooper C and Sayer AA. (2006a) Developmental origins of midlife physical performance: evidence from a British birth cohort. American Journal of Epidemiology, 164, 110-21.

Kuh D, Hardy R, Butterworth S, Okell L, Wadsworth M, Cooper C. and Aihie Sayer A. (2006b) Developmental origins of midlife grip strength: findings from a birth cohort study. The Journals of Gerontology Series A, Biological Sciences and Medical Sciences, 61, 702-6.

Leversen KT, Sommerfelt K, Ronnestad A, Kaaresen PI, Farstad T, Skranes J, Stoen R, Elgen IB, Rettedal S, Eide GE, Irgens LM and Markestad T. (2010) Predicting neurosensory disabilities at two years of age in a national cohort of extremely premature infants. Early Human Development, 86, 581-6.

Montgomery SM. (2010) Coordination, childhood weight gain and obesity. Canadian Medical Association Journal, 182, 1157-8.

Olsson GM, Hulting AL and Montgomery SM. (2008) Cognitive function in children and subsequent type 2 diabetes. Diabetes Care, 31, 514-6.

Osika W and Montgomery SM. (2008) Physical control and coordination in childhood and adult obesity: Longitudinal Birth Cohort Study. British Medical Journal, 337, a699.

Osika W, Montgomery SM, Dangardt F, Wahrborg P, Gan LM, Tideman E and Friberg P. (2009) Anger, depression and anxiety associated with endothelial function in childhood and adolescence. Archives of Disease in Childhood. doi:10.1136/adc.2008.152777

PASW. ( 2009) http://www.spss.com/uk/statistics/

Stenberg AE, Wang H, Fish J, Schrott-Fischer A, Sahlin L and Hultcrantz M. (2001) Estrogen receptors in the normal adult and developing human inner ear and in Turner's syndrome. Hearing Research, 157, 87-92.

Stenhammar C, Olsson GM, Bahmanyar S, Hulting AL, Wettergren B, Edlund B and Montgomery SM. (2010) Family stress and BMI in young children. Acta Paediatrica, 99, 1205-12.

The NS, Adair LS and Gordon-Larsen P. (2010) A study of the birth weight-obesity relation using a longitudinal cohort and sibling and twin pairs. American Journal of Epidemiology, 172, 549-57.

Tong S, Baghurst P, Vimpani G and McMichael A. (2007) Socioeconomic position, maternal IQ, home environment, and cognitive development. Journal of Pediatrics, 151, 284-8.

Welch D and Dawes PJ. (2007) Childhood hearing is associated with growth rates in infancy and adolescence. Pediatric Research, 62, 495-8.

WHO 1995. Physical Status: The Use and Interpretation of Anthropometry

WHO Technical Report Series. Geneva, WHO.

Yaffe K, Blackwell T, Kanaya AM, Davidowitz N, Barrett-Connor E and Krueger K. (2004a) Diabetes, impaired fasting glucose, and development of cognitive impairment in older women. Neurology, 63, 658-63.

Yaffe K, Kanaya A, Lindquist K, Simonsick EM, Harris T, Shorr RI, Tylavsky FA and Newman AB. (2004b) The metabolic syndrome, inflammation, and risk of cognitive decline. Journal of the American Medical Association, 292, 2237-42 
